\title{
F. H. Bradley entre orthodoxie et hérésie : le sens des 'Concluding Remarks' de Ethical Studies
}

F. H. Bradley Between Orthodoxy and Heresy: the Meaning of the 'Concluding Remarks' of Ethical Studies

Jean-Paul Rosaye

\section{OpenEdition}

Journals

Édition électronique

URL : http://journals.openedition.org/rfcb/3652

DOI : $10.4000 /$ rfcb.3652

ISSN : 2429-4373

Éditeur

CRECIB - Centre de recherche et d'études en civilisation britannique

Édition imprimée

Date de publication : 1 mars 2013

ISBN : 2-911580-37-0

ISSN : 0248-9015

Référence électronique

Jean-Paul Rosaye, «F. H. Bradley entre orthodoxie et hérésie : le sens des 'Concluding Remarks' de Ethical Studies », Revue Française de Civilisation Britannique [En ligne], XVIII-1 | 2013, mis en ligne le 01 mars 2013, consulté le 22 mars 2020. URL : http://journals.openedition.org/rfcb/3652 ; DOI : https:// doi.org/10.4000/rfcb.3652

Ce document a été généré automatiquement le 22 mars 2020.

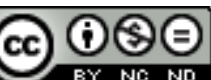

Revue française de civilisation britannique est mis à disposition selon les termes de la licence Creative Commons Attribution - Pas d'Utilisation Commerciale - Pas de Modification 4.0 International. 


\title{
F. H. Bradley entre orthodoxie et hérésie : le sens des 'Concluding Remarks' de Ethical Studies
}

\author{
F. H. Bradley Between Orthodoxy and Heresy: the Meaning of the 'Concluding \\ Remarks' of Ethical Studies
}

Jean-Paul Rosaye

1 Si Ethical Studies (1876) marque la première intervention remarquée de Francis Herbert Bradley sur la scène philosophique anglaise de son temps, les 'Concluding Remarks' par lesquelles il clôt cet ouvrage n'ont pas reçu toute l'attention qu'elles méritent. Ce n'est pas faute d'avoir été lues, ni d'avoir fait date dans l'histoire des idées de l'époque ; on peut même ajouter qu'elles ont été très importantes pour Bradley au regard de son évolution personnelle, philosophique et religieuse. Cette situation aurait peut-être dû susciter un intérêt plus marqué de la part des commentateurs, qui ont trop hâtivement soutenu la thèse d'une négligence de l'œuvre bradleyenne pendant un bon demi-siècle. Une lecture attentive de ce texte permet en effet d'éclairer en partie les raisons de ce qui apparaît aujourd'hui encore comme un véritable malentendu philosophique, et de faire droit à certaines de ses positions les plus critiquées.

2 Après une première lecture superficielle, il est déjà possible de constater l'ambiguïté des 'Concluding Remarks' : une marque de fabrique, diront les spécialistes de Bradley. Mais si cette ambiguïté porte sur la façon dont il a exposé ses idées, on peut en dire autant des interprétations qui en ont été faites: son texte a été tenu pour ce qu'il n'était pas, et il a été interprété dans le sens du temps, en dépit de certaines prises de position très hétérodoxes. En réalité, comme nous nous proposons de le démontrer, ces «remarques conclusives " se présentent comme un miroir de la situation religieuse et philosophique en Grande-Bretagne au moment où Bradley pensait qu'il était devenu nécessaire de réapprendre à la philosophie à parler anglais.

3 Le message diffusé dans les 'Concluding Remarks' aura été entendu: Bradley a été perçu comme un «sage de la foi », et ses 'Concluding Remarks' ont été lues comme un 
texte fondamental par certains de ses contemporains, notamment les membres de la London Ethical Society ${ }^{1}$. Il est vrai qu'il y réaffirmait la centralité de la foi et l'importance de la religion, dans un contexte où l'agnosticisme s'imposait de plus en plus; et cela était au moins aussi attendu que la justification de la nécessité qu'il y avait d'insérer l'individu dans la matrice collective de la moralité, à une époque où la chose éthique était devenue un enjeu de civilisation.

\section{Une apparence d'orthodoxie}

4 Les 'Concluding Remarks' fournissent le terme donnant son sens au mouvement de Ethical Studies, un ouvrage assez difficile du fait de ses analyses nouvelles et originales, qui révèle la complexité de problèmes qu'on avait jusque-là cru simples, le tout déroulé au fil d'une dialectique serrée : elles mettent fin à toutes les impressions fâcheuses qui auraient pu apparaître en éclairant définitivement l'ensemble de son propos. En effet, sans nécessairement en rabattre sur les prétentions de l'ouvrage, sans forcer le discours dans un sens qui contreviendrait à la vérité, Bradley revient dans cette conclusion à des propos plus familiers. C'est, nous semble-t-il, la fonction que joue ce que l'on pourrait appeler un premier niveau de lecture de ces 'Concluding Remarks'.

5 La conscience humaine a été présentée dans les deux derniers essais de Ethical Studies comme le siège d'un conflit intérieur entre le soi (self) et le soi idéal (ideal self), et cela vaut autant pour la conscience morale que pour la conscience religieuse, à ceci près que le soi idéal, dans le cas de la conscience religieuse, est un réel (c'est-à-dire que la réalisation du soi idéal dans la moralité est effective), et qu'il s'agit d'un conflit entre deux volontés, l'une humaine, rongée par le péché, et l'autre divine: comme l'écrit Bradley, aucune image n'est suffisamment forte pour représenter les tourments auxquels donne lieu la contradiction ressentie de leur inadéquation ${ }^{2}$. Le point capital ici est que ce conflit se produit à l'intérieur du sujet : les deux volontés, l'humaine et la divine, sont internes au sujet, et non pas séparées sur le mode d'un sujet intérieur et d'un objet extérieur. L'internalité des deux volontés permet en effet d'expliquer la conscience du péché, la rébellion contre Dieu, mais aussi le désir de Dieu, le besoin de grâce et la possibilité de l'expiation ${ }^{3}$. Mais comme la conscience n'a pas l'intuition permanente que la volonté divine est la volonté de son soi le plus intime, il en résulte un sentiment de séparation avec Dieu, un sentiment de son extériorité qui éclaire le fait que le divin soit posé comme objet.

6 Le véritable problème consiste dès lors à découvrir comment se réconcilier avec cette volonté qui n'est pas humaine ; c'est tout le problème de la foi, et de la justification par la foi qu'expose alors Bradley en insistant sur la valeur indépassable du protestantisme dans l'effectuation de cette reconnaissance :

You must believe that you too really are one with the divine, and must act as if you believed it. In short, you must be justified not by works but solely by faith. This doctrine, which Protestantism, to its eternal glory, has made its own and sealed with its blood, is the very centre of Christianity; and where you have not this in one form or another, there Christianity is nothing but a name. ${ }^{4}$

7 À la lecture de ces pages et de celles qui suivent, il apparaît que Bradley est orthodoxe vis-à-vis du dogme anglican de la justification par la foi seule et de l'idée que les œuvres, par lesquelles s'exprime la volonté humaine, participent également de la justification, dans la mesure où ce sont des œuvres de la foi ${ }^{5}$. Sa position est aussi 
paulinienne. En expliquant que l'homme doit abdiquer sa volonté propre pour s'anéantir dans la volonté divine, il utilise à dessein toute la thématique paulinienne de la mort glorieuse ${ }^{6}$.

L'objet avec lequel le soi est unifié par la foi est réel pour la foi, d'une part, parce que le cours du monde est la réalisation de la Volonté divine et, d'autre part, parce que, sur le plan intérieur, l'humain et le divin sont Un : la foi soutient qu'il existe « un royaume de Dieu » (a kingdom of God), un organisme qui se réalise dans ses membres. La foi pratique est donc bien la finalité, et le culte ou les exercices de piété ne sont religieux que s'ils renforcent la volonté religieuse, s'ils sont au service de l'esprit, et non de la lettre, s'ils visent la réconciliation, c'est-à-dire reflètent la Volonté divine en l'homme, ou, et c'est la phrase finale de Ethical Studies, s'ils permettent une fusion dans l'unité avec Dieu grâce à la médiation de l'amour? .

9 Toute paulinienne également est l'adhésion manifeste des 'Concluding Remarks' à la conception de l'homo spiritualis que l'on trouve en 1 Cor 2: 14-15, où saint Paul oppose l'homme naturel à l'homme spirituel. La foi est incompatible avec la connaissance sensuelle immédiate ${ }^{8}$, ou quelque connaissance de type naturel, même si toute certitude théorique n'exclut pas la foi: la connaissance religieuse n'est pas une connaissance ordinaire, et c'est la raison pour laquelle Bradley insiste ici encore sur le fait que la foi ne porte pas sur ce qui est "visible ", que la connaissance véritable ne passe pas par les voies habituelles de la connaissance et de la représentation. La foi, explique Bradley, n'est ni une simple croyance, ni un acte théorique du jugement, ni une "vision ", qui l'exclut"; la connaissance naturelle, comme la sagesse, ne suffit pas pour la foi, qui n'est pas un problème de degré mais de nature de connaissance, et c'est la raison pour laquelle l'objet religieux ne fait pas partie du monde visible ${ }^{10}$.

10 La vision de l'homme et de la société qui est exposée renvoie à un modèle qui rappelle la distinction qu'opérait saint Augustin entre la cité terrestre et la cité de Dieu. En éprouvant les limites de la moralité et en jaugeant la condition humaine, on doit postuler la nécessité d'une sphère religieuse possédant la vérité profonde de la moralité ${ }^{11}$, et poser l'existence d'une Église véritable, transcendant les églises temporelles des communautés religieuses : 'Religious communities may be called "churches"; but churches in this sense must not be confounded with the Church proper. That is the whole body of Christ' ${ }^{12}$. Un rapport de similitude est entretenu avec la totalité relative de l'organisme politique, à ceci près que ce qui était visible et fini dans ce dernier est invisible et infini dans le royaume de Dieu. De fait, l'État et la religion sont indissolublement liés, et la religion constitue l'horizon indépassable de l'État. Si des devoirs subsistent dans la religion, ils ne sont pas du même ordre que ceux de la moralité en ce qu'ils sont intérieurs, et visent un ordre du cœur, ce qui est l'esprit même du Nouveau Testament ${ }^{13}$.

11 Dans ces 'Concluding Remarks' se trouvent donc incontestablement des éléments consensuels. De surcroit, comme s'il voulait se prémunir contre une lecture désobligeante, Bradley multiplie les remarques destinées à désamorcer une critique éventuelle ; c'est volontairement, compte tenu de l'objet même de l'ouvrage, qu'il omet d'aborder des questions essentielles dans cette conclusion modeste, et si le lecteur s'inquiétait de certaines absences, malgré le fond d'évidence qui semble animer l'auteur sur ce qui pourrait être la vraie religion, Bradley ajoute : 'We are to keep to a minimum, and the reader must not conclude that we repudiate whatever we say nothing about. ${ }^{14}$ 


\section{Un texte peu orthodoxe, en réalité}

12 Le second niveau de lecture, qui concerne un public moins large que le précédent, demande que soit dressé un bilan de ce qu'il en est, au bout du compte, de la nature et des rapports de la morale, de la religion et de la philosophie. C'est celui où réside véritablement l'originalité de la pensée bradleyenne, ou, pour le dire autrement, celui où apparaissent les libertés qu'il prend avec les orthodoxies, tant par l'absence d'éléments attendus que par la présence d'autres, qui peuvent être jugés peu souhaitables.

13 Tout d'abord, Bradley conduit des attaques contre un certain type de religiosité sur plus d'une demi-douzaine de pages. En effet, en dépit de l'importance que certaines pratiques peuvent avoir dans la vie religieuse, ni l'apprentissage de la doctrine, ni le fait d'aller à l'église, ni la méditation, ni la prière, ni les exercices de dévotion, etc. ne sont des activités religieuses en soi, car, même si elles peuvent aider à mettre sur la voie d'une attitude religieuse correcte, si elles sont des médiations permettant d'intensifier et de renforcer la moralité, elles peuvent aussi se faire prendre au piège de l'unilatéralité et dégénérer en esthétisme, voire en irréligion ${ }^{15}$. C'est la raison pour laquelle l'aspect public, voire politique, de la religion, n'est jamais que secondaire par rapport à la véritable religion :

You can have true religion without sacraments or public worship, and again both without clergymen; just as you can have clergymen and sacraments without true religion. And if some of the clergy think that they stand in a more intimate relation with the divine Spirit than the rest of the community do, then they both go against the first principles of Christianity. ${ }^{16}$

14 On peut se demander quel rôle jouent de telles remarques. Cela peut certes participer d'une défense classique de l'esprit contre la foi, d'un refus de tout philistinisme. Cela peut, pour qui voudra s'adonner à des exercices de psychanalyse spontanée, renvoyer à l'enfance de l'auteur et à ses rapports douloureux avec le rigorisme religieux et moral de son père. Mais plus encore, cela nous semble renvoyer à une prise de position consciente dans le débat de l'époque: les lecteurs que de tels propos choqueront, Bradley veut les choquer; les lecteurs pour lesquels de tels propos seront une évidence sont les lecteurs auxquels Bradley veut s'adresser.

Dès son premier ouvrage, The Presuppositions of Critical History, publié deux ans avant Ethical Studies, Bradley a choisi la science contre les combats d'arrière-garde que mène un certain fanatisme religieux. L'histoire critique a le droit d'étudier les textes bibliques, l'évolution darwinienne est un fait, la philosophie doit intégrer, interpréter, amender les résultats de la science, et non pas interdire ses progrès. Quant à la critique du dogmatisme et des prétentions des autorités religieuses, Bradley appartient au même monde que celui de Huxley. Bien entendu, cela ne signifie pas que, comme Huxley, Bradley choisisse l'autorité de la science contre celle de l'Église : la philosophie conserve tous ses droits, et le dogmatisme scientifique en vaut un autre... D'ailleurs, les convictions philosophiques de Huxley sont mises à mal par la critique plus qu'ironique que Bradley conduit vis-à-vis de Matthew Arnold, un auteur dont Huxley a subi l'influence. Bradley analyse et ridiculise notamment l'idée selon laquelle la religion est une "moralité teintée d'émotion" et du moralisme au petit pied ${ }^{17}$, ainsi que la conception arnoldienne de la culture : tout ce qu'a pu écrire Arnold sur la religion n'est que du «baratin littéraire» ('literary clap-trap'18). Ainsi, ne serait-ce que pour réduire 
les prétentions d'une postérité qui ne lui a pas rendu justice, nous dirons que Bradley n'est pas seulement un homme de son temps : en matière de liberté de l'esprit, il fait aussi partie de l'avant-garde - et qu'il ne perd, par de tels propos, que des lecteurs qui ne pourront jamais le suivre.

Mais Bradley ne se contente pas de prendre congé d'une conception dogmatique de la religion, il prend aussi des distances vis-à-vis de l'orthodoxie, distances dont il nous faut maintenant prendre la mesure. Ainsi, il ne manifeste pas beaucoup d'intérêt pour ce qui précède le Nouveau Testament. Critiquant la conception arnoldienne de la culture impliquant une lecture historique de la religion chrétienne, il s'élève contre la prétention de certains passages de l'Ancien Testament à rendre compte de la réalité ${ }^{19}$, estime que la religion chrétienne n'a plus à se reconnaître dans les pratiques hébraïques anciennes ${ }^{20}$, puisque faire de l'observance d'une loi l'alpha et l'oméga d'une religion est une erreur : "It is the belief "with the heart" that is wanted; and where that is not religion is not. ${ }^{21}$ Bien sûr, Bradley insiste sur la dimension protestante, le libre examen, le rôle de la foi seule, mais il y a plus que cela. D'abord une lecture hégélienne du christianisme implique que l'on fasse de ce dernier, et même plus exactement du protestantisme, la vérité de la religion, dont les formes antérieures sont moins vraies. Une lecture empruntée à David Friedrich Strauss de ce même christianisme fait prendre beaucoup de distance vis-à-vis de ce qui relève d'un corpus mythicolégendaire, même si cela ne doit pas nécessairement venir à l'encontre de toute religiosité. Enfin, la référence, d'une part, au corpus grec dans toute l'analyse de la moralité, dont la religion est l'apothéose, plutôt qu'à des textes plus anciens, et, d'autre part, à l'œuvre de saint Paul, qui a commencé à intégrer la philosophie grecque dans le christianisme, justifie, sans même que l'on évoque des traces de marcionisme, que l'on se prenne à douter de la validité du premier niveau de lecture.

Le deuxième point qu'il convient de noter est une absence significative. Dans toutes les 'Concluding Remarks', il n'est fait allusion qu'en deux points à un élément qui puisse renvoyer à la Trinité : dans une note, Bradley cite Jacob Boehme, dont le caractère parfaitement orthodoxe n'est pas le trait le plus marquant, et cette citation utilise le terme "Christ " ${ }^{22}$; le terme revient dans une autre note ${ }^{23}$, à propos d'un point technique sur le culte et un problème de définition des églises. Mais Bradley n'évoque à aucun moment la centralité de l'Incarnation dans le message chrétien. À aucun moment, le terme de Père n'est utilisé, mais seulement celui de Dieu. À aucun moment, l'EspritSaint n'est nommé. On dira que dans la communion de l'Église invisible, il est implicitement présent, mais, d'une part, l'implicite n'est pas l'explicite, et, d'autre part, cette totalité organique relève d'une interprétation hégélienne marquée qui ne laisse guère de place au lexique traditionnel. Cependant, le premier niveau de lecture laissait en place suffisamment d'éléments consensuels pour qu'un terme trinitaire puisse se glisser. Visiblement, le souci phénoménologique de Bradley l'aura conduit à s'interdire impitoyablement ces écarts de langage. Enfin, il faut s'interroger sur la nature de la foi telle que l'entend Bradley. Elle implique, nous l'avons vu, une montée de la volonté humaine vers la volonté divine, une ascension de la pensée vers une identification et une unification qui est la véritable réalisation de soi comme tout infini :

Faith implies the rise in thought, but not that only; it implies also the rise of the will to the object, which is not seen but thought. And this presupposes the practical separation for me of myself and the object. In the mere theoretic rise I do not think of myself, but only of the object : in faith I must also have myself before me [...] Faith then is the recognition of my true self in the religious object, and the 
identification of myself with that both by judgement and will [...] Justification by faith means that, having thus identified myself with the object, I feel myself in that identification to be already one with it, and enjoy the bliss of being, all falsehood overcome, what I truly am..$^{24}$

18 À le dire ainsi, rien ne semble en désaccord avec l'orthodoxie. Mais il faut aller plus avant et se demander comment cette ascension a lieu. Le point de départ, c'est la moralité, telle qu'elle se met en place chez le jeune enfant, telle qu'elle se réalise imparfaitement dans la cité terrestre : la religion prend son origine dans l'insuffisance de l'État, dans une société où l'art et la science, par exemple, produisent des conflits d'interprétation et de devoirs ${ }^{25}$. La conscience religieuse est le seul lieu possible de l'accomplissement final :

In the very essence of the religious consciousness we find the relation of our will to the real ideal self. We find ourselves, as this or that will, against the object as the real ideal will, which is not ourselves, and which stands to us in such a way that, though real, it is to be realised, because it is all and the whole reality. ${ }^{26}$

19 Mais quelles sont les forces qui permettent cet accomplissement à l'intérieur même de l'esprit de l'homme? De quelle nature est la relation qui se joue entre la volonté humaine et la volonté de Dieu? Fort classiquement, la religion chrétienne fait intervenir ici le concept de grâce. Mais ici encore, une recherche minutieuse ne permet de trouver qu'une occurrence du terme de "grâce ", malgré de très nombreuses analyses de la foi ${ }^{27}$.

Le moins que l'on puisse dire, c'est que la question de la grâce n'est pas centrale, puisqu'elle n'est ici qu'un des nombreux éléments du lexique religieux classique qui perd tout sens si l'analyse psychologique que livre Bradley n'est pas accordée, et qui est la seule chose qui importe ici, puisqu'elle engage toute la conception métaphysique des rapports entre le sujet et l'objet, comme le démontre la longue note qui lui est accolée. Or, si l'on s'en tient à cette analyse psychologique et métaphysique, à l'ascension (le terme 'rise' est constamment utilisé) du sujet vers sa vérité, le tout infini auquel il aspire, force est de constater que cette sotériologie équivoque est bien un effort du sujet qui remonte à sa véritable origine naturelle dans une perspective grecque et néoplatonicienne. À aucun moment, il n'est expliqué que cette dialectique ascendante s'appuie sur autre chose que ce dont le sujet fini dispose par nature. À aucun moment l' atonement ne semble dépendre d'autre chose que de ce 'Good will' qui comprend son propre mouvement constitutif. Comment donc mettre fin à la contradiction sentie qui est au commencement de l'homme, si ce n'est de l'animal? Là encore, on ne saurait aller jusqu'à taxer Bradley de pélagianisme, mais l'absence de référence à la lutte entre le péché et la grâce est fort curieuse dans l'étude de la religion, même ramenée à la conscience religieuse ${ }^{28}$. Toute réalisation ultime, toute réconciliation provient pour Bradley d'un effort de la volonté, d'un travail intérieur. Et si Ethical Studies se clôt par une évocation de l'amour éternel et par une citation de Pétrone, ce n'est pas seulement parce que l'épicurisme de ce dernier constitue une pique contre l'utilitarisme : elle met en lumière l'idée du vrai plaisir, comme pour marquer une bonne fois pour toutes où se situe la vraie compréhension du bonheur humain.

21 Mais l'analyse de Bradley ne manifeste pas seulement des absences significatives, elle s'appuie aussi sur des sources positives. Bien que soucieux de ne pas encombrer le lecteur par une érudition superflue, il n'hésite pas à reconnaître sa dette majeure vis-àvis de Wilhelm Vatke ${ }^{29}$. Ce dernier, presque oublié aujourd'hui, a proposé dans les années 1840 une solution intéressante à la problématique mise en place par Hegel 
lorsqu'il avait distingué la philosophie et la religion non pas par leur contenu, mais par une différence de forme, la religion restant au niveau de la représentation. Cette distinction a été l'occasion de la rupture entre hégéliens de gauche et de droite, les seconds restant fidèles à la distinction hégélienne, tandis que les premiers la prenaient comme prétexte pour en rabattre sur les prétentions de la religion: David Strauss, Jeune Hégélien et ami proche de Vatke, avait, dans son ouvrage célèbre sur la vie de Jésus, grandement porté atteinte à la vérité traditionnelle de la religion. Il n'est pas possible ici d'entrer dans les méandres de cette querelle, mais elle était fondée indéniablement sur une difficulté de la doctrine hégélienne. En effet, en bonne logique dialectique, la distinction entre la forme et le contenu n'est pas valide, et relève de l'entendement: il manque une médiation, que certains théologiens ont tenté de trouver ${ }^{30}$. La solution de Vatke consistait à démontrer que si la religion était dépassée par la philosophie dans l'ordre théorique, où, effectivement, elle relevait de la représentation, en revanche dans l'ordre pratique, qui, en quelque sorte, était son domaine réservé, elle conservait toute son importance. C'est à la mise en œuvre de cette solution que Vatke se livre dans l'ouvrage cité par Bradley, qui démontre ici, d'une part, une connaissance fine du contexte germanique, alliée à une bonne maitrise des textes de l'idéalisme allemand ; d'autre part, une véritable autonomie dans le choix de ses sources d'inspiration, puisqu'il parvient à découvrir, dans un foisonnement polémique tout de même considérable, les ouvrages qui l'aident à manifester sa pensée en construction selon des directions qu'il a déjà définies. Ainsi, Bradley retient de Vatke sa détermination de la sphère de la religion en rapport avec celle de la moralité, sa distinction dans l'accomplissement de cette dernière des deux totalités qui l'expriment (celle de la communauté étatique et celle de la communauté ecclésiale), son étude de la nature de la volonté, son analyse de la nécessité métaphysique du mal comme condition de réalisation du bien, toutes choses par lesquelles Vatke reste fidèle, si ce n'est parfaitement à la lettre, du moins à l'esprit de l'hégélianisme, qu'il pense n'avoir qu'à parachever. Deux éléments méritent d'être remarqués au sujet de Vatke, qui ne reçoivent pas de Bradley un soutien clair et enthousiaste, sans que ce dernier ne dise vraiment où portent les points de désaccord. C'est ce qu'il nous faut essayer de faire en rapportant l'ouvrage allemand à Ethical Studies.

Le premier consiste dans l'absence, pour Vatke, de Dieu véritablement personnel : il en fournit une démonstration que des contemporains autorisés ont reconnue et attribuée à une forme de néo-platonisme ${ }^{31}$. Le second point consiste dans l'évolution de Vatke : sa distinction d'une sphère pratique spécifique à la religion, dans le cadre du système hégélien, devait être instable, puisqu'il a fini par adhérer à une forme proche de celle de Schleiermacher, l'ennemi juré de Hegel. Nous retrouvons Bradley sur ce point, car, contrairement à Vatke, dont l'hégélianisme se voulait orthodoxe, force est de constater que notre auteur avait déjà rompu avec un élément essentiel de la pensée hégélienne, à savoir la place de la philosophie dans un futur système métaphysique.

Dans ses 'Concluding Remarks', Bradley ne manque pas seulement à l'orthodoxie religieuse, il manque aussi à l'orthodoxie hégélienne. Car s'il emprunte à Vatke son analyse de la sphère de la moralité et de la religion, il la détache totalement du système dont elle ne constitue plus alors un moment nécessaire, mais une sphère existant de façon autonome, et dont la consistance est même indéniablement supérieure à celle de la philosophie. Car la religion, qui est une sortie de la dialectique incluse dans le devoirêtre, vise l'être, le réellement réel, et l'atteint : 
Religion is essentially a doing, and a doing which is moral. It implies a realising, and a realising of the good self. Are we to say then that morality is religion? Most certainly not. In mere morality the ideal is not: it for ever remains a "to be". The reality in us or the world is partial and inadequate; and no one could say that it answers to the ideal, that, morally considered, both we and the world are all we ought to be, and ought to be just what we are. We have at furthest the belief in an ideal which in its pure completeness is never real; which, as an ideal, is a mere "should be". ${ }^{2}$ volonté humaine et le réel s'effectue ${ }^{33}$, et non pas dans d'autres activités humaines affectées d'une insuffisance fondamentale, comme l'art et la science ${ }^{34}$. En effet, que ce soit dans l'art, dans la philosophie et la religion, le soi, explique Bradley, parce qu'il se sent comme envahi par la vérité et la beauté, tente d'objectiver obscurément la plénitude qu'il ressent et de la reconstruire, c'est-à-dire la réaliser : 'And in this, so far, art, philosophy, and religion are the same ${ }^{35}$. Leur différence dans cette " activité " provient de ce que la volonté humaine n'est pas de l'ordre de l'essence dans l'art et la philosophie, et que, même si ces dernières activités évoquent la relation de la volonté humaine à l'idéal réel, elles demeurent des activités théoriques et des représentations dont le processus consiste à aboutir à un résultat visible, extérieur, distinct, qui ne sert qu'à démontrer la cohérence de cette volonté, à la clarifier, à se poser des questions sur ce qu'elle vise, mais jamais à la considérer en tant que telle dans sa relation à la réalité ${ }^{36}$. Si l'art, la religion et la philosophie sont les trois moments de la constitution du savoir absolu dans l'Encyclopédie de Hegel, Bradley non seulement en intervertit l'ordre dans sa conclusion, mais refuse également à la philosophie, qui n'est donc pas savoir absolu, le pouvoir d'accéder véritablement au réel, au titre qu'il s'agit là d'une exclusivité de la religion. Chez Bradley, il n'existe pas de savoir de Dieu, mais uniquement une relation par laquelle il est saisi, éprouvé dans l'expérience, "réalisé » dans le contact intérieur qui se produit en soi, dans une communion par réconciliation, par atonement. Certes, Hegel est trop présent dans Ethical Studies pour que l'on puisse prétendre qu'il a été balayé d'un revers de la main. Il convient plutôt de dire qu'il a permis une transition vers une philosophie des premiers principes que Bradley appelle de ses vœux, et qui ne pouvait se constituer sans son apport.

En fin de compte, et aussi importante que soit sa dette vis-à-vis de Hegel, puisque c'est encore à des hégéliens comme Vatke qu'il emprunte ses moyens de s'éloigner de Hegel, il nous semble impossible de ne pas considérer la tradition idéaliste insulaire, dans sa dimension néo-platonicienne, comme le véritable terreau sur lequel se développe la pensée de Bradley. D'ailleurs, qui, parmi les idéalistes de la période de Jowett et de Green a gardé le cap d'un idéalisme néo-hégélien pur? Ce n'est pas le cas de Jowett ni de Green, et force est de constater que Bradley se situe également dans leur sillage. Il est alors possible de relier la pensée bradleyenne à la tradition mystique du platonisme chrétien, que R.W. Inge a appelé le troisième type de pensée et de croyance chrétiennes dans ses Hulsean Lectures (1926).

\section{Conclusion}

Les 'Concluding Remarks' semblent avoir rasséréné les lecteurs de l'époque quant à la possibilité d'inscrire dans un mouvement philosophique anglais une opposition à l'expansion de l'utilitarisme, du matérialisme et de l'agnosticisme ambiants, ainsi que 
la mise à distance d'une philosophie étrangère dont la puissance théorique risquait de masquer la profondeur de la vision religieuse de l'humanité. De ce point de vue, l'accent sur la justification par la foi seule et la glorification des valeurs du protestantisme, les références marquées à saint Paul, à saint Augustin et au Nouveau Testament, le refus de la spéculation théorique abstraite autant que du scientisme, sans pour autant, loin s'en faut, refuser la science, peuvent, à première vue, apparaître consensuels et justifier la valeur de l'ouvrage de Bradley dans le cadre d'un combat de l'idéalisme pour s'imposer de nouveau en Angleterre. Certainement, la délimitation claire d'un espace spéculatif et contemplatif se situant au-delà de toute valorisation possible dans un contexte de "révolution des valeurs $»^{37}$ a contribué à offrir au mouvement idéaliste dans son ensemble une position à partir de laquelle il était possible de réfléchir. Mais cette lecture rassurante esquive un certain nombre d'éléments qui imposent de travailler encore en direction d'une métaphysique capable de réduire les développements contradictoires qu'en l'état, on est en droit de craindre. Dans son article sur ce qu'il estimait être la doctrine centrale de Bradley dans Ethical Studies, l'idée de self-realization, David Crossley écrivait que Bradley était parvenu à une position dangereuse, aux frontières de la philosophie, de la religion et du mysticisme en utilisant la philosophie hégélienne ${ }^{38}$. Le système que Bradley conçoit après Ethical Studies, du fait de son appartenance à deux modes de pensée sur la façon de caractériser le réel, l'une hégélienne et l'autre d'inspiration platonicienne, ne pouvait véritablement contenter ni les hégéliens, ni les mystiques.

Certes, l'intention qui a présidé à l'écriture de Ethical Studies n'a pas consisté à satisfaire les uns et les autres, mais à parcourir justement les frontières de la philosophie et de la religion à la recherche d'une solution éthiquement acceptable dans un monde irrésistiblement tourné vers les promesses de la science.

\section{NOTES}

1. Ian MACKILLOP, The British Ethical Societies, Cambridge: Cambridge University Press, 1986, p. 83.

2. F. H. BRADLEY, Ethical Studies, Oxford: Clarendon Press, 1962 (seconde édition revue et corrigée de 1927, avec une introduction de Richard Wollheim), p. 323.

3. Bradley interprète le terme 'atonement' comme une « réconciliation », mais il reconnaît aussi la possibilité d'utiliser d'autres expressions pour en rendre compte (voir Ibid., p. 324).

4. Ibid., p. 325.

5. Ibid., p. 329.

6. Ibid., p. 325. Bradley commente longuement la « mort » du 'this me', du soi privé, jusqu'à la page 329 , où il admet de façon explicite sa lecture paulinienne. Il semble qu'il se réfère ici essentiellement à 2 Cor 6:9, mais aussi à Rom 3:20, 23-24 pour l'impossibilité de toute autojustification.

7. Ibid., p. 342.

8. Ibid., p. 327.

9. Ibid.

10. Ibid. 
11. Voir, par exemple, saint Augustin, La cité de Dieu, 3 vols., Paris : Seuil (Points Sagesses), 1982, vol. 3, p. 110 (liv. XIX, sect. VI) ; p. 128 (liv. XIX, sect. XVII).

12. BRADLEY, op. cit., p. 339 , note 1 .

13. Ibid., p. 337.

14. Ibid., p. 330.

15. Ibid., pp. 337-338.

16. Ibid., pp. 339-340.

17. Ibid., pp. 318, note 2 .

18. Ibid., pp. 317-318.

19. Ibid., p. 317.

20. 'To those of us who do not think that Christianity is called upon to wrap itself any longer in "Hebrew old clothes", all this is entirely a matter for the historian' (Ibid., p. 317). L 'expression 'Hebrew old clothes ' est de Carlyle.

21. 'By denying such a doctrine we ought not to give offense to the Christians. Whether we shall give offense or not is another matter. We are sorry if it is so; but nevertheless we deny the assertion, and we think that on our side we have the religious consciousness and the New Testament.' (Ibid., pp. 336-337)

22. Ibid., p. 328.

23. Ibid., p. 339.

24. Ibid., pp. 327-328.

25. 'The practical content which religion carries out comes from the state, society, art, and science' (Ibid., p. 333).

26. Ibid., p. 320.

27. Ibid., p. 323.

28. La référence fréquente de Bradley à un livre de Wilhelm Vatke, Die Menschliche Freiheit in ihrem Verhältniss zur Sünde und zur Göttliche Gnade, se fait systématiquement sous le titre, Die Menschliche Freiheit, alors que son objet majeur est bien la dialectique du péché (Sünde) et de la grâce (Gnade)...

29. 'Throughout the sequel I have to acknowledge my indebtedness to Vatke's book, Die menschliche Freiheit, 1841' (BRADLEY, op. cit., p. 314). Voir également pp. 293, 298, 299.

30. Jahn Rohls a étudié ce moment de la théologie dans "Die Aufhebung der religiösen Vorstellung in den philosophischen Begriff, Hegel These und die Theologie der Junghegelianer », in Ingolf U. DALFERTH \& Hans-Peter GROSSHANS (eds.), Kritik der Religion: Zur Aktualität einer unerledigten philosophischen und theologischen Aufgabe, Mohr Siebeck, 2006. Il a crédité Vatke d'une tentative de résolution qui n'est pas en désaccord avec Hegel et qui vise à rétablir l'unité du savoir et de la foi, sans préjudice pour aucun des deux.

31. Voir Heinrich BENECKE, Wilhelm Vatke in seinem Leben und seinen Schriften, Bonn, 1883, p. 347.

32. BRADLEY, op. cit., p. 315.

33. Ibid., p. 319.

34. Ibid., p. 320.

35. Ibid.

36. Ibid., p. 321.

37. 'The individual life for religion is one with the divine; it possesses infinite worth, a value no terms can express' (Ibid., p. 334).

38. David J. CROSSLEY, 'Self-Realization as Perfection in Bradley's Ethical Studies', Idealistic Studies, vol. 7, n³ 3, septembre 1977, p. 212. 


\section{RÉSUMÉS}

Les 'Concluding Remarks' de F. H. Bradley parachevant Ethical Studies (1876) sur une apothéose de la foi ont été peu étudiées par la critique sur l'auteur en dépit de leur influence certaine sur les sociétés éthiques de la période victorienne tardive et de leur importance dans la suite de son œuvre. Face à la montée du matérialisme et de l'agnosticisme, Bradley a suggéré dans ce texte une solution religieuse ambiguë à la question éthique. En cherchant à consolider le concept de réalisation de soi pour affermir son opposition aux visions de l'éthique véhiculées par l'utilitarisme et le kantisme, Bradley a proposé une interprétation de la foi oscillant entre orthodoxie et hérésie qui ne pouvait contenter qu'un nombre réduit de fidèles.

F. H. Bradley's 'Concluding Remarks', ending his Ethical Studies (1876), were undeniably significant in prompting his subsequent metaphysical work. Yet, they have rarely been studied as a separate text, despite its fame among the members of the London Ethical Society in lateVictorian times. In this text, Bradley's answer to the threat of materialism and agnosticism endangering true faith and orthodoxy shaped a very ambiguous religious solution to ethics. While consolidating the concept of self-realization he had contributed to define against utilitarian and Kantian presuppositions, his views suggested an interpretation of faith wavering between an orthodox statement and some heretical positions which could satisfy but a small number of religious believers.

\section{AUTEUR}

\section{JEAN-PAUL ROSAYE}

Université d'Artois 